\title{
Flow Patterns Transition Law of Oil-Water Two-Phase Flow under a Wide Range of Oil Phase Viscosity Condition
}

\author{
Wei Wang, Wei Cheng, Kai Li, Chen Lou, and Jing Gong \\ Beijing Key Laboratory of Urban Oil \& Gas Distribution Technology, Department of Mechanical and Transportation Engineering, \\ China University of Petroleum, Beijing 102249, China
}

Correspondence should be addressed to Jing Gong; ydgj@cup.edu.cn

Received 16 July 2013; Accepted 13 September 2013

Academic Editor: Bo Yu

Copyright (c) 2013 Wei Wang et al. This is an open access article distributed under the Creative Commons Attribution License, which permits unrestricted use, distribution, and reproduction in any medium, provided the original work is properly cited.

A systematic work on the prediction of flow patterns transition of the oil-water two-phase flows is carried out under a wide range of oil phase viscosities, where four main flow regimes are considered including stratified, dispersed, core-annular, and intermittent flow. For oil with a relatively low viscosity, VKH criterion is considered for the stability of stratified flow, and critical drop size model is distinguished for the transition of o/w and w/o dispersed flow. For oil with a high viscousity, boundaries of core-annular flow are based on criteria proposed by Bannwart and Strazza et al. and neutral stability law ignoring that the velocity of the viscous phase is introduced for stratified flow. Comparisons between predictions and quantities of available data in both low and high viscosity oilwater flow from literatures show a good agreement. The framework provides extensive information about flow patterns transition of oil-water two-phase flow for industrial application.

\section{Introduction}

Oil-water two-phase systems abound in many applications in the petroleum industry including emulsions preparation, oilwater mixture separation, and transportation. Accurate prediction of oil-water flow characteristics, such as flow pattern transition, is important in many engineering applications. Despite the importance, however, oil-water flow has not been explored to the same extent as gas-liquid flow. The density difference between the two phases is relatively small, while the viscosity ratio encountered can extend over several orders of magnitude, both of which bring great complexities and difficulties to the study of oil-water flow transitions.

Various experimental works have been proposed in the literature to enhance the understanding of oil-water flow transitions [1-4] in horizontal pipes with the flow pattern being classified as stratified, dispersed, and mixed flow. Trallero [4] conducted extensive experimental and theoretical studies on oil-water flow patterns, where segregated and dispersed flow were mainly focused and a total of six patterns were identified.

For the transition law of the flow patterns, Brauner and Maron [5] first developed a two-fluid model to characterize the transition from stratified flow to other flow patterns. Trallero [4] proposed that VKH criterion could predict the transition between stratified flow and unstable wavy stratified flow. Brauner [6] suggested an approach for the transition to dispersed flow pattern. Recently, Sharma et al. [7] introduced the energy minimization concept, a principle that a system can be stabilized to its minimum total energy, into oil-water flow pattern transition.

Most of the published works focus on low viscosity oilwater flows. However, when the viscosity of oil increases, new characteristics could be observed. For heavy oil-water dispersions, core-annular flow, especially oil core-water annular flow, becomes dominant. Moreover, drop entrainment as well as oil clot and slug can easily be found in such flow.

For the transition of core-annular flow pattern, Bannwart [8] argued that core-annular flow is prone to occur in a pipe where the two fluids have very dissimilar viscosities but relatively close densities. In this case, however, it is proposed that velocity difference of the two phases should be small. Grassi et al. [9] found that the extension of theoretical models for low viscosity ratio to high viscosity ratio flows should not be straightforward. Rodriguez et al. [10] developed a refined 
pressure-loss prediction model which includes a slip ratio term that implicitly accounts for the buoyancy of the oil core.

From the previous literature, it could be gained that systematic work for the prediction of flow pattern transition is still demanded. This paper aims to establish a framework for flow pattern transition prediction especially over a wide range of oil viscosity, which is expected to provide extensive support for the industrial application of oil-water flow pattern transition theories. Results are validated with a wide range of existing published data, even that of heavy crude oils.

\section{Theoretical Models of Flow Pattern Transition}

2.1. Flow Pattern Classification. Although large amounts of experiments on oil-water two-phase flow in horizontal pipe have been conducted by scholars, there still lacks overall consensus about how the flow patterns could be classified. This may be due to the fact that experimental conditions differ and ways to observe flow regimes are not identical. In spite of that, the common regimes without drop entrainment are mainly considered here. Combine Taitel and Dukler's gas-liquid flow pattern classification method with oil-water pipe flow characteristics, the flow patterns of low viscosity oil-water two-phase flow are classified as stratified flow, w/o dispersed flow, o/w dispersed flow, and intermittent flow.

\subsection{Transition of Stratified Flow}

2.2.1. Transition of Stratified Flow for Low Viscosity Ratio. Recent researches of stratified flow stability are all based on momentum and continuity equation of double fluid model by introducing the influence of small disturbance on the interface. It is common that ways of treating the stability equations could be classified into two categories: ZNS (zero neutral stability)/ZRC (zero real characteristics) criterion and KelvinHelmholz (IKH and VKH) instability theory. Brauner and Moalem Maron [11] used ZNS/ZRC criterion to predict the transition criterion of smooth stratified flow pattern to other flow patterns. ZNS line is set as the boundary of smooth stratified and stratified wavy and ZRC line as the upper limit of smooth stratified. Trallero [4] argued that the VKH equation determines the transition between stable stratified flow and either unstable stratified with wavy interface or some other flow patterns and may be used to predict onset of entrainment, which is suitable for low viscosity oil-water systems. The equations are as follows:

$$
\begin{aligned}
& \overbrace{\left(C_{\mathrm{V}}-C_{\mathrm{IV}}\right)^{2}+\underbrace{\frac{\rho_{\mathrm{w}} \rho_{\mathrm{o}}}{\rho H_{\mathrm{w}} H_{\mathrm{o}}}\left(U_{\mathrm{w}}-U_{\mathrm{o}}\right)^{2}-\underbrace{}_{\mathrm{i}} \frac{\left(\rho_{\mathrm{w}}-\rho_{\mathrm{o}}\right) g \cos \beta A}{\rho S_{\mathrm{i}}}-\frac{\sigma A}{\rho S_{\mathrm{i}}} k^{2}}_{\text {IKH }}+\frac{\rho_{f}\left(U_{\mathrm{w}}-U_{\mathrm{o}}\right)^{2} C_{S} A}{\rho}\left(\frac{1}{A_{\mathrm{w}}}-\frac{1}{A_{\mathrm{o}}}\right)}^{\mathrm{VKH}}<0 \\
& \overbrace{J_{\mu}+\underbrace{J_{U}-J_{g}-J_{\sigma}}_{\text {IKH }}+J_{s}}^{\mathrm{VKH}}<0 \\
& C_{\mathrm{V}}=\frac{\partial F / \partial H_{\mathrm{w}}}{\partial F / \partial U_{\mathrm{so}}-\partial F / \partial U_{\mathrm{sw}}} \\
& F=-\frac{\tau_{\mathrm{w}} S_{\mathrm{w}}}{A_{\mathrm{w}}}+\frac{\tau_{\mathrm{o}} S_{\mathrm{o}}}{A_{\mathrm{o}}}+\tau_{\mathrm{i}} S_{\mathrm{i}}\left(\frac{1}{A_{\mathrm{w}}}+\frac{1}{A_{\mathrm{o}}}\right)=0 \\
& C_{\mathrm{IV}}=\frac{\rho_{\mathrm{w}} U_{\mathrm{w}} H_{\mathrm{w}}+\rho_{\mathrm{o}} U_{\mathrm{o}} H_{\mathrm{o}}}{\rho_{\mathrm{w}} H_{\mathrm{w}}+\rho_{\mathrm{o}} H_{\mathrm{o}}} \\
& \rho=A\left(\frac{\rho_{\mathrm{w}}}{A_{\mathrm{w}}}+\frac{\rho_{\mathrm{o}}}{A_{\mathrm{o}}}\right) \text {, }
\end{aligned}
$$

where $\rho_{\mathrm{w}}$ and $\rho_{\mathrm{o}}$ are the density of water and oil, respectively; $H_{\mathrm{w}}$ and $H_{\mathrm{o}}$ are the holdup of water and oil, respectively; $U_{\text {sw }}$ and $U_{\text {so }}$ are superficial velocity of water and oil, respectively; $U_{\mathrm{w}}$ and $U_{\mathrm{o}}$ are real mean velocity of water and oil, respectively; $\tau_{\mathrm{w}}, \tau_{\mathrm{o}}$, and $\tau_{\mathrm{i}}$ are water, oil, and interfacial shear stresses; $S_{\mathrm{w}}, S_{\mathrm{o}}$, and $S_{\mathrm{i}}$ are water, oil, and interface wet perimeter; $A_{\mathrm{w}}, A_{\mathrm{o}}$, and $A$ are cross-sectional area of water, oil, and the pipe; $C_{\mathrm{V}}$ and $C_{\mathrm{IV}}$ are interface wave velocity and critical interface wave velocity, and $k$ is the wave number.

The first item on the left of (1) is the viscous instability, depending on $\tau_{\mathrm{w}}, \tau_{\mathrm{o}}$, and $\tau_{\mathrm{i}}$; the second item is the velocity instability, related to velocity difference of the two phases; the third item is the gravity stability; the fourth item is interface tension stability, related to interface tension and interface wave number; the fifth is a correction term which stands for the influence of other interface instable factors. $C_{S}$ is the correction coefficient.

In order to apply the stratified flow criterion, the in-situ water and oil velocities as well as cross-section parameters must be resolved firstly as in-situ water and oil velocities as well as cross-section parameters can be regarded as a function of liquid level, $h_{\mathrm{w}}$. 
Fairuzov [12] found that, while solving the steady state parameters of (4) for gas-liquid horizontal stratified flow, there may appear more than one solution, but, for oil-water stratified flow, there exists only one solution in the global scope. Therefore, in the current study, liquid level $h_{\mathrm{w}}$ is obtained by varying the parameter from 0 to $D$.

2.2.2. Transition of Stratified Flow for High Viscosity Ratio. In derivation of the neutral stability equation of stratified liquidliquid flow, Brauner [13] found that the lower fluid of stratified flow is sometimes much faster, so the velocity of the upper phase may be neglected in neutral stability condition. Such an extreme situation may be of practical relevance, for example, in the transportation of high viscosity oil-water system. Ignoring the time and space variations in the upper fluid velocity yields

$$
\begin{gathered}
U_{\mathrm{sw}}^{2}\left(\frac{C_{\mathrm{rn}}}{U_{\mathrm{w}}}-1\right)^{2}<\frac{16}{\pi^{2}} \frac{D \widetilde{A}_{\mathrm{w}}^{3}}{\rho_{b} \widetilde{A}_{\mathrm{w}}^{\prime}}\left(\Delta \rho g+\sigma k^{2}\right) \\
C_{\mathrm{rn}}=\frac{U_{\mathrm{w}}\left(\partial F / \partial U_{\mathrm{w}}\right)-\left(A_{\mathrm{w}} / A_{\mathrm{w}}^{\prime}\right)(\partial F / \partial H)}{\left(\partial F / \partial U_{\mathrm{w}}\right)},
\end{gathered}
$$

where $C_{\mathrm{rn}}$ is the real neutral stable wave velocity.

2.3. Transition of Dispersed Flow. Dispersed flow exists on the condition that the continuous phase is turbulent and the turbulent kinetic energy in the whole region is sufficiently intense to break the dispersed droplets into drops smaller than the critical diameter:

$$
\begin{gathered}
d_{\max }<d_{\text {cr }}, \quad \operatorname{Re}_{C} \geq 2100, \\
1.82 \operatorname{Re}_{C}^{-0.7}<\frac{d_{\text {crit }}}{D}<0.1,
\end{gathered}
$$

where $D$ is the inner diameter of the pipe.

2.3.1. Phase Inversion Point. It is important to introduce the phase inversion point. Factors that influence phase inversion vary, including two-phase density, viscosity, interfacial tension, and other physical properties and operation conditions. At the same time, temperature, oil-water system formation, and mixture container wettability also play an impact. The inversion point aims to provide a probable range of distinguishment between continuous and disperse phase where Decarre and Fabre [14] model is introduced:

$$
\varphi_{\text {inv }}=\left[1+\left(\frac{\mu_{\mathrm{o}}}{\mu_{\mathrm{w}}}\right)^{1 / 6}\left(\frac{\rho_{\mathrm{o}}}{\rho_{\mathrm{w}}}\right)^{1 / 6}\right]^{-1},
$$

where $\varphi_{\text {inv }}$ stands for water content of inverting point.

2.3.2. Maximum Diameter. In the turbulent field, the dispersed droplet is dominately affected by the turbulent inertia shear. Therefore, the maximum stable drop is the balance between turbulent kinetic energy and droplet interface energy [6]:

$$
\begin{gathered}
\mathrm{W} e_{\text {crit }}=\frac{\tau d_{\max }}{\sigma}, \\
\frac{\rho_{c} u^{\prime 2}}{2} \approx \frac{4 \sigma}{d_{\max }} .
\end{gathered}
$$

In Chen's work [15], maximum drop size of dilute oilwater dispersed flow depends on the energy dissipation rate of the continuous phase and is directly proportional to $\left(\varepsilon^{-0.4}\right)$. Meanwhile, by introducing Barnea's [16] idea of gas-liquid bubbly flow, the dispersed volume fraction is taken into consideration, which gives

$$
\begin{gathered}
d_{\max }=\left(1+a \varphi^{s}\right)\left(\frac{\rho_{c}}{\rho_{d}}\right)^{0.33}\left(\frac{\sigma}{\rho_{c}}\right)^{0.6} \varepsilon^{-0.4}, \\
\varepsilon=\frac{2 f U_{m}^{3}}{D},
\end{gathered}
$$

where $\varepsilon$ is the energy dissipation rate, $U_{m}$ is the mixture velocity, $f$ is the friction coefficient, and the subscripts $c$ and $d$ stand for continuous phase and dispersed phase, respectively.

The higher the concentration of the dispersed phase, the more the energy needed by the continuous phase. Brauner [6] supposed that the incoming flow of the continuous phase should carry sufficient turbulent energy to disrupt the tendency to coalescence and to disperse the other phase. So the maximum droplet diameter can be expressed as

$$
\begin{aligned}
d_{\max }= & 2.22 C_{H}^{3 / 5}\left(\frac{\rho_{c} U_{c}^{2} D}{\sigma}\right)^{-0.6} \\
& \times\left(\frac{\varepsilon_{\mathrm{w}}}{1-\varepsilon_{\mathrm{w}}}\right)^{0.6}\left[\frac{\rho_{m}}{\rho_{c}\left(1-\varepsilon_{\mathrm{w}}\right)} f\right]^{-0.4} D,
\end{aligned}
$$

where $C_{H}$ is a constant, $C_{H}=O(1) \cdot \varepsilon_{\mathrm{w}}$ is the water holdup. $\rho_{m}$ is the density of mixture.

2.3.3. Critical Diameter. Due to the gravity or buoyancy effect, droplets will be separated from the mainstream, migrating to the wall. Considering the equilibrium of gravity and turbulent forces only, the droplet of critical dimension can be obtained through

$$
\frac{d_{c b}}{D}=\frac{3 \rho_{c} f U_{c}^{2}}{8\left|\rho_{c}-\rho_{d}\right| D g} .
$$

By assuming the buoyancy force is strong enough to overcome interfacial tension and break a dispersed drop, the critical size can be expressed through the balance between interfacial tension and buoyancy forces:

$$
\frac{d_{c \sigma}}{D}=\left[\frac{0.4 \sigma}{\left|\rho_{c}-\rho_{d}\right| g D^{2}}\right]^{0.5} .
$$


The practical critical diameter is the minimum between the two conditions including oil-water two-fluid system and operation condition:

$$
\frac{d_{\text {crit }}}{D}=\operatorname{Min}\left(\frac{d_{c \sigma}}{D}, \frac{d_{c b}}{D}\right)
$$

2.4. Transition of Core-Annular Flow. Joseph et al. [17] used the hydrodynamic stability theory and concluded that a fully developed laminar-laminar core flow having the same density could stay stable when more viscous fluid is placed at the core and occupies most of the cross-section. Considering the stability of a viscous liquid flowing in the core, which was surrounded by a turbulent annulus, Bannwart [8] extended the previous model by using effective viscosity:

$$
\mu_{\mathrm{o}, \mathrm{eff}}>\mu_{\mathrm{w}, \mathrm{eff}}, \quad \mu_{\mathrm{o}}>\mu_{\mathrm{w}}+0.0005 \rho_{\mathrm{w}} U_{\mathrm{sw}} D
$$

where $\mu_{\text {eff }}$ is the effective viscosity which is the sum of the absolute molecular viscosity with the turbulent viscosity. To obtain the predicted transition boundary, it is necessary to find the critical $U_{s w}$. Through a two-fluid model, Brauner [13] proposed an expression for calculating the core diameter, $D_{c}$, as a function of $U_{s w}$ and $U_{\text {so }}$, given the setup conditions and fluid properties. Considering a laminar core-turbulent annular flow, the expression that includes the influence of drop entrainment is given as

$$
\begin{gathered}
\frac{D_{c}}{D}=\left\{\phi \times\left(\chi \phi\left[\frac{\mu_{\mathrm{o}}}{\mu_{\mathrm{w}}} \frac{\rho_{\mathrm{w}}}{\rho_{b}}\left(\frac{\nu_{\mathrm{w}}}{\nu_{b}}\right)^{2} \frac{G(\phi)^{1.8}}{F(\phi)}\right]^{1 / 2}\right.\right. \\
\left.\left.+\phi+\frac{G(\phi)}{F(\phi)}\right)^{-1}\right\}^{1 / 2},
\end{gathered}
$$

where $\phi=U_{\text {so }} / U_{\text {sw }}$ and $\chi$ is the Lockhart-Martinelli parameter which is fully based on superficial variables:

$$
\begin{aligned}
& F(\phi)=\frac{\alpha_{\mathrm{w}} \phi-\left(1-\alpha_{\mathrm{w}}\right)}{\phi\left(\alpha_{\mathrm{w}}+\alpha_{c}-1\right)}, \\
& G(\phi)=\frac{\alpha_{c}-\left(1-\alpha_{c}\right) \phi}{\alpha_{\mathrm{w}}+\alpha_{c}-1},
\end{aligned}
$$

where $\alpha_{\mathrm{w}}$ and $\alpha_{c}$ stand for water volume fraction in the annular and oil volume fraction in the core. Once the oil superficial velocity $U_{\text {so }}$ and $H_{\mathrm{o}}=H_{\mathrm{o}}$ are fixed, the critical water velocity corresponding to the transition from core-annular flow is obtained.

\section{Results and Discussion}

3.1. Model Diagram. The oil-water flow pattern transition prediction is based on sound results of fluid properties, certain pipelines, and operation parameters. Thus the criterion can be integrated as given in Figure 1.
Figure 2(a) indicates how the diameter affects the stability of the stratified flow and dispersed flow. The diameters are 1,2, and 3 inches, respectively. Viscosity ratio of oil to water $(\widetilde{\mu})$ is 29.6 and interface tension is $0.036 \mathrm{~N} / \mathrm{m}$. Prediction indicates that the scope of stratified flow becomes broader when the diameter increases, but the scope of $\mathrm{o} / \mathrm{w}$ and w/o dispersed flow becomes narrower. It can be concluded that, for oil-water two-phase flows, stratified flow pattern is more likely to form in a large-diameter pipe, as the effect of gravity stable term (1) is more obvious, which, to a certain extent, hinders the growth of interface wave and stabilizes the two-phase flow while, in smaller pipes, dispersed oil-water flow is relatively common, due to the fact that flow energy dissipation rate and turbulent kinetic energy are large though mixing velocity is unchanged. In addition, under the same diameter and internal phase concentration, the velocity needed to maintain a complete $\mathrm{o} / \mathrm{w}$ dispersed flow pattern is bigger than that of a complete w/o dispersed flow pattern.

Figure 2(b) shows the influence of viscosity on stability of stratified and dispersed flow. The viscosities are 10, 50, and $80 \mathrm{mPa} \cdot \mathrm{s}$, respectively, while pipe diameter is $2 \mathrm{inch}$, and the interfacial intension is $0.036 \mathrm{~N} / \mathrm{m}$. Prediction indicates that the scope of stratified flow becomes narrower with larger oil viscosity while the scope of w/o dispersed flow becomes broader, and the sensitivity of o/w dispersed flow boundary is weaker.

The more viscous the oil, the stronger the influence of viscous instability and the smaller the superficial velocity needed to maintain the two phases stratified. From (9) and (12), dispersed viscosity plays no impact on maximum droplet diameter and critical droplet diameter. Therefore, for o/w dispersed flow, the model does not provide the trend of boundary changes with oil viscosity. This is due to the present model neglecting the effect of dispersed drop viscosity.

3.2. Discussions. Models are validated against available experimental data. Two cases of low viscosity oil (viscosity ratio of oil/aqueous phase $<100$ ) and two higher ones (viscosity ratio $>100$ ) are selected and compared. Comparisons with Oglesby [1] and Trallero [4] results are chosen as a representation of low viscosity.

Figure 3(a) is a comparison between predicted results and Trallero [4] experimental data (viscosity ratio 29.6), which indicates a good agreement between the predicted results of $\mathrm{o} / \mathrm{w}$ and $\mathrm{w} / \mathrm{o}$ transition boundaries and the experimental data. The scope of stratified flow with mixed interface (stratified wavy flow) in experiment is very large; the proper explanation is that the low viscosities of both phases make it easier for drop entrainment to take place. Actually this flow pattern is a transitional flow pattern from stratified flow to dispersed flow. The predicted transition boundary of stratified flow includes some stratified flows with mixed interface.

Figure 3(b) compares the predicted boundaries with the experimental data from Oglesby [1] (viscosity ratio 62.8). The prediction of w/o boundary fits well with experimental data. The prediction of $\mathrm{o} / \mathrm{w}$ dispersed flow boundary is narrower than experimental results. This is partly because the sensitivity of the o/w dispersed flow criterion is weak (discussed above). Another explanation is that oil and water were 


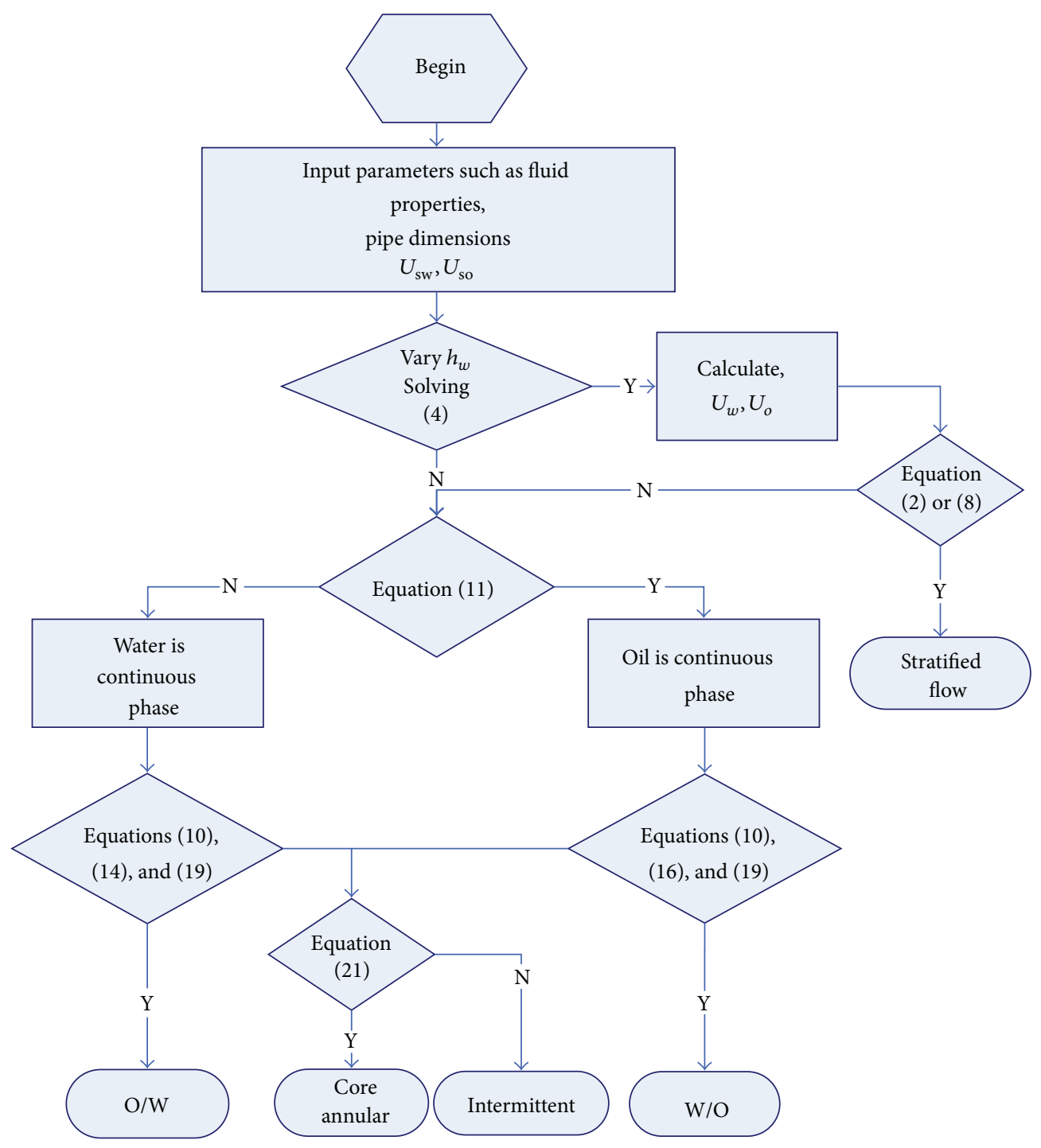

FIgURE 1: Diagram of flow pattern transition program.

mixed before entering the experimental system. The premix process makes $\mathrm{o} / \mathrm{w}$ dispersed flow scope larger and stratified flow scope narrower. In addition, the predicted scope of stratified flow is narrow, and this is because the viscous instability becomes stronger.

Figure 4(a) is a comparison between predicted results and Yao and Gong [18] experimental data (viscosity ratio 325.4), while Figure 4(b) compares the predicted boundaries and experimental data from Wang et al. [19] (viscosity ratio 620). Core-annular, stratified, w/o dispersed, and intermittent flow are considered.

Both figures show that the stratified flow transition criterion fail to predict the accurate region. This may be caused by drop entrainment in viscous oil. And pure single phase in certain region of the pipe cross-section rarely exists. It should be noted that (7) does not take into account the drop entrainment in both phases, whose regime actually is Dw/o and Do/w. In addition, the core-annular/Do/w transition boundary agrees well with experimental data. This boundary is the lowest oil holdup requirement, beyond which the oil core will be broken and finally dispersed in the water. As water drop is easily entrained into the viscous oil phase, the flow regime of Dw/o becomes dominant when phase inversion occurs. Phase inversion point can be perfectly regarded as a criterion for Dw/o transition boundary.

\section{Conclusions}

In current work, theoretical models concerning systems with both low and high viscosity ratio are introduced for criterion of flow pattern transition of oil-water horizontal flow. The main flow regimes considered include water stratified flow, core-annular flow, w/o dispersed flow, o/w dispersed flow, and intermittent flow. A systematic work is carried out for flow pattern transition with oil viscosity varying in a wide range.

It is concluded that stability of the oil-water stratified flow in horizontal pipe is strongly related to oil viscosity, gravity, 


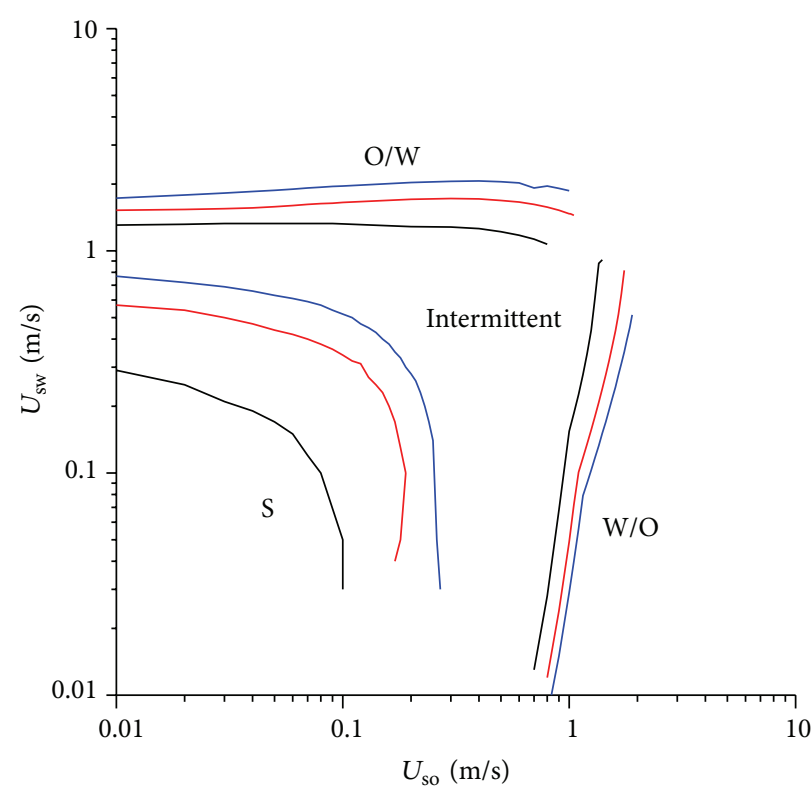

$\widetilde{\rho}=0.85, \widetilde{\mu}=29.6, \sigma=0.036 \mathrm{~N} \cdot \mathrm{m}$

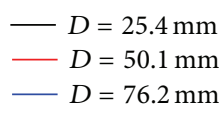

(a) Pipe diameter

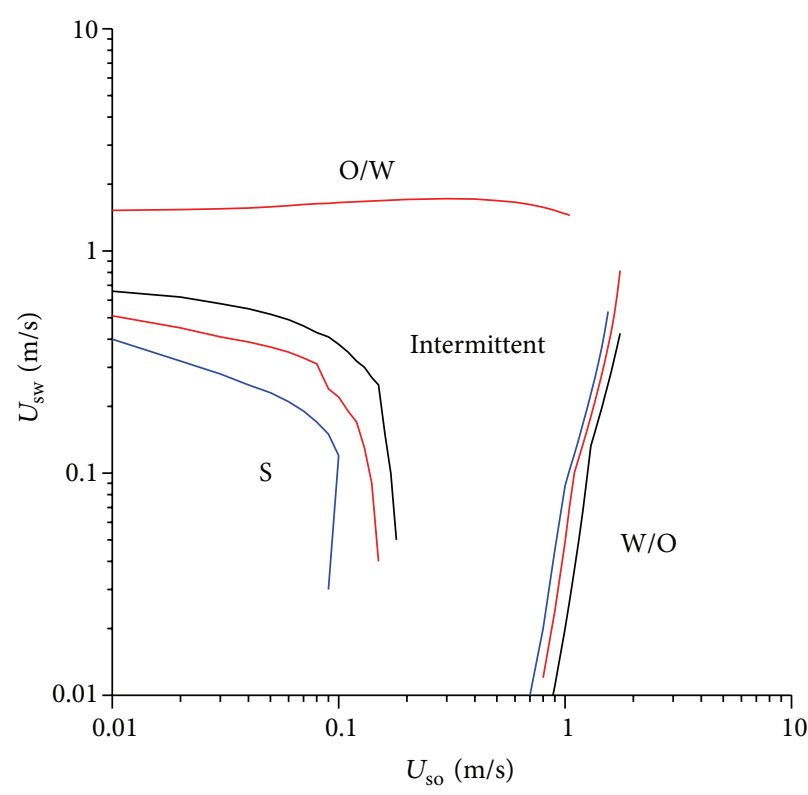

$\tilde{\rho}=0.85, \sigma=0.036 \mathrm{~N} \cdot \mathrm{m}, D=50.1 \mathrm{~mm}$

$\begin{aligned}-\mu & =10 \mathrm{mPa} \cdot \mathrm{s} \\ -\mu & =50 \mathrm{mPa} \cdot \mathrm{s} \\ \mu & =80 \mathrm{mPa} \cdot \mathrm{s}\end{aligned}$

(b) Oil phase viscosity

Figure 2: Prediction of flow pattern transition.

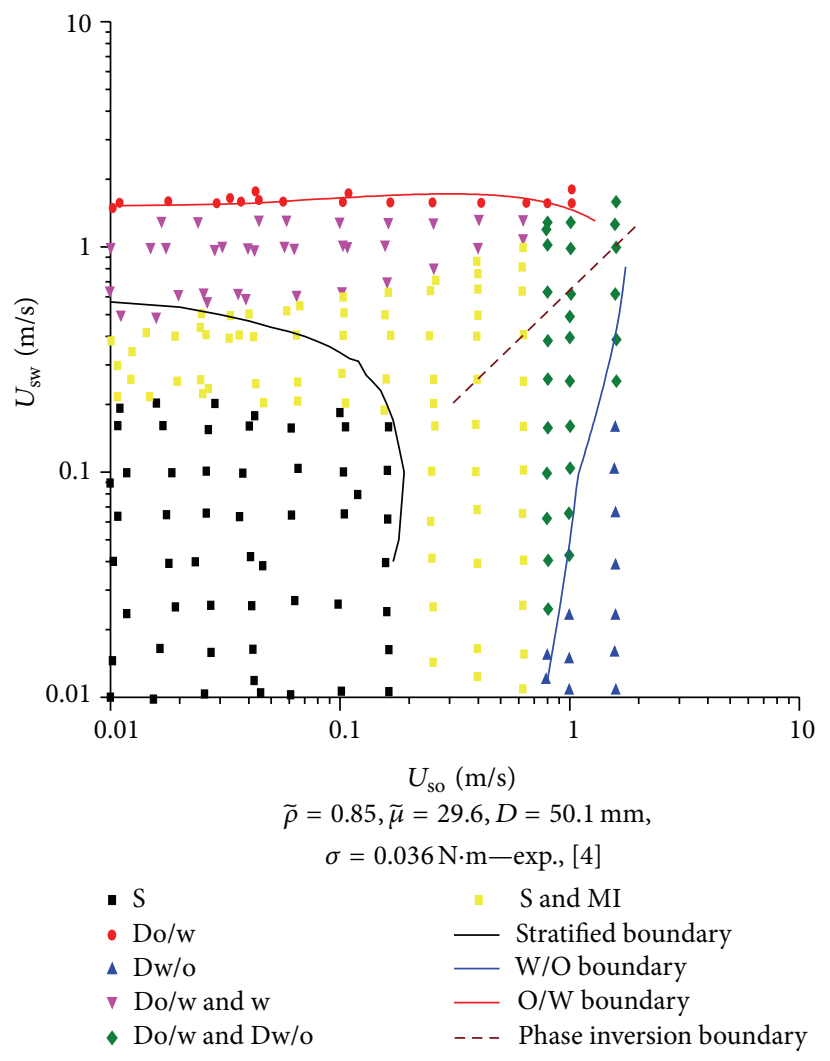

(a)

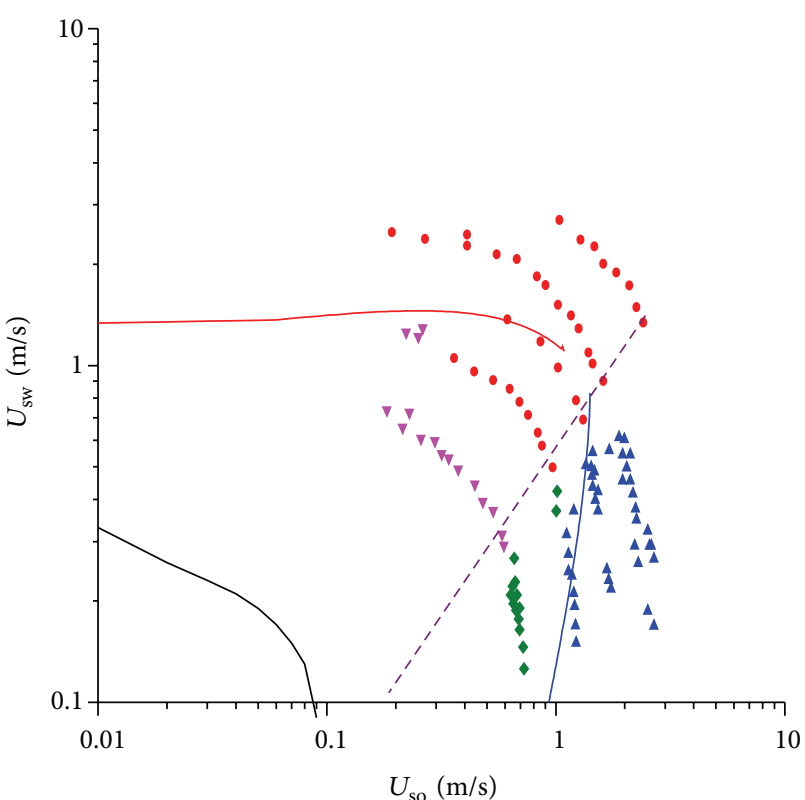

$\widetilde{\rho}=0.86, \widetilde{\mu}=61.0, \sigma=0.0294 \mathrm{~N} \cdot \mathrm{m}$,

$D=40.6 \mathrm{~mm}$-exp., [1]

- Do/w
Dw/o
Do/w and w
Do/w and Dw/o

- W/O boundary

- O/W boundary

- - - Phase inversion boundary

_ Stratified boundary

(b)

FIgURE 3: Comparison of experimental data (symbols) and prediction (lines) of oil-water two phase flow pattern transition. 


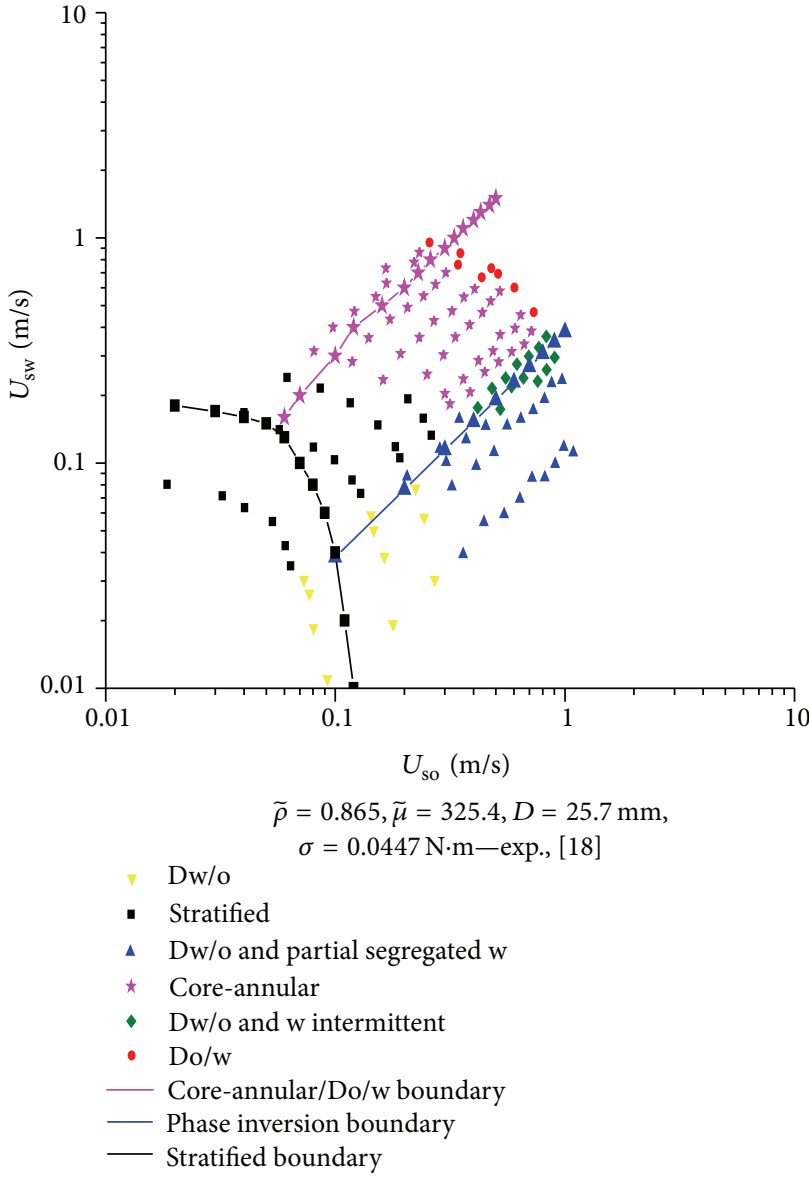

(a)

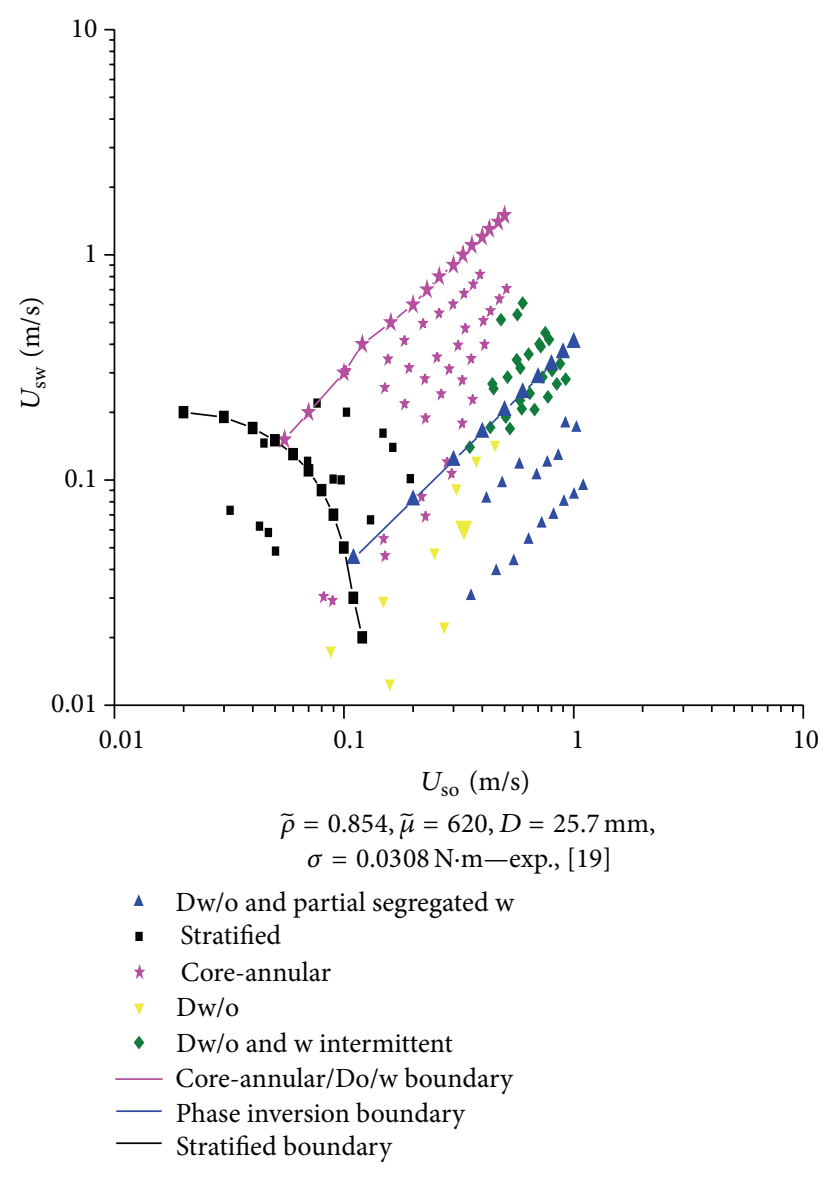

(b)

FIGURE 4: Comparison between prediction (lines) and experimental data (symbols) of oil-water flow.

and interfacial intension. For viscous oils, the influence of shear stress becomes much more obvious and can be characterised by ignoring the velocity of the viscous phase while, in dispersed flow, as the viscosity increases, oil droplets become harder to breakup, meaning that the ability of droplets to recover deformation becomes stronger. It is more difficult for $\mathrm{o} / \mathrm{w}$ dispersed flow to be formed in viscosity oil. Additionally, core-annular flow tends to occur in a pipe where the two fluids have much different viscosities but relatively close densities. Drop entrainment occurs easily in core-annular flow and must be taken into consideration in the transition criterion.

\section{Acknowledgments}

The authors wish to thank the National Natural Science Foundation of China (51104167), the program for New Century Excellent Talents in University (NCET-12-0969), the Foundation for the Author of National Excellent Doctoral Dissertation of PR China (201254), the National Science and Technology Specific Program of China (2011ZX05026-004-03), and the Foundation of China University of Petroleum-Beijing (BJ-2011-02) for the financial support for this work.

\section{References}

[1] K. D. Oglesby, An experimental study on the effects of oil viscosity, mixture velocity and water fraction on horizontal oil water flow [M.S. thesis], The University of Tulsa, Tulsa, Okla, USA, 1979.

[2] S. Arirachakaran, K. D. Oglesby, M. Malinowsky, M. S, O. Shoham, and J. P. Brill, "An analysis of oil water flow phenomena in horizontal pipes," in Proceedings of the SPE Production Operations Symposium, 1989.

[3] T. Al-Wahaibi and P. Angeli, "Transition between stratified and non-stratified horizontal oil-water flows. Part I: stability analysis," Chemical Engineering Science, vol. 62, no. 11, pp. 2915-2928, 2007.

[4] J. L. Trallero, Oil-water flow pattern in horizontal pipes [Ph.D. thesis], The University of Tulsa, Tulsa, Okla, USA, 1995.

[5] N. Brauner and D. M. Maron, "Two phase liquid-liquid stratified flow," Physicochemical Hydrodynamics, vol. 11, no. 4, pp. 487-506, 1989.

[6] N. Brauner, "The prediction of dispersed flows boundaries in liquid-liquid and gas-liquid systems," International Journal of Multiphase Flow, vol. 27, no. 5, pp. 885-910, 2001.

[7] A. Sharma, A. Al-Sarkhi, C. Sarica, and H. Zhang, "Modeling of oil-water flow using energy minimization concept," International Journal of Multiphase Flow, vol. 37, no. 4, pp. 326-335, 2011. 
[8] A. C. Bannwart, "Modeling aspects of oil-water core-annular flows," Journal of Petroleum Science and Engineering, vol. 32, no. 2-4, pp. 127-143, 2001.

[9] B. Grassi, D. Strazza, and P. Poesio, "Experimental validation of theoretical models in two-phase high-viscosity ratio liquidliquid flows in horizontal and slightly inclined pipes," International Journal of Multiphase Flow, vol. 34, no. 10, pp. 950-965, 2008.

[10] O. M. H. Rodriguez, A. C. Bannwart, and C. H. M. de Carvalho, "Pressure loss in core-annular flow: modeling, experimental investigation and full-scale experiments," Journal of Petroleum Science and Engineering, vol. 65, no. 1-2, pp. 67-75, 2009.

[11] N. Brauner and D. Moalem Maron, "Stability analysis of stratfied liquid-liquid flow," International Journal of Multiphase Flow, vol. 18, no. 1, pp. 103-121, 1992.

[12] Y. V. Fairuzov, "Stability analysis of stratified oil/water flow in inclined pipelines," SPE Production and Facilities, vol. 16, no. 1, pp. 14-21, 2001.

[13] N. Brauner, "Two-phase liquid-liquid annular flow," International Journal of Multiphase Flow, vol. 17, no. 1, pp. 59-76, 1991.

[14] S. Decarre and J. Fabre, "Phase inversion behavior for liquidliquid dispersions," Revue de l'Institute Francais du Petrole, vol. 52, no. 4, pp. 415-424, 1997.

[15] J. Chen, Experimental study on horizontal oil water pipe flow [Ph.D. thesis], China University of Petroleum, Beijing, China, 2003.

[16] D. Barnea, "Transition from annular flow and from dispersed bubble flow-unified models for the whole range of pipe inclinations," International Journal of Multiphase Flow, vol. 12, no. 5, pp. 733-744, 1986.

[17] D. D. Joseph, M. Renardy, and Y. Renardy, "Instability of the flow of immiscible liquids with different viscosities in a pipe," Journal of Fluid Mechanics, vol. 141, pp. 309-317, 1984.

[18] H. Y. Yao and J. Gong, "An Experimental investigation on flow patterns and pressure gradient of heavy oil-water flows in horizontal pipes," Advances in Multiphase Flows, vol. 2, pp. 359-361, 2004.

[19] W. Wang, J. Gong, and P. Angeli, "Investigation on heavy crudewater two phase flow and related flow characteristics," International Journal of Multiphase Flow, vol. 37, no. 9, pp. 1156-1164, 2011. 


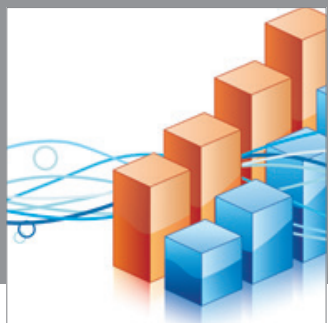

Advances in

Operations Research

mansans

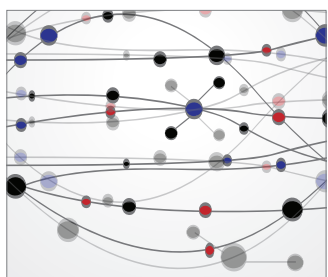

The Scientific World Journal
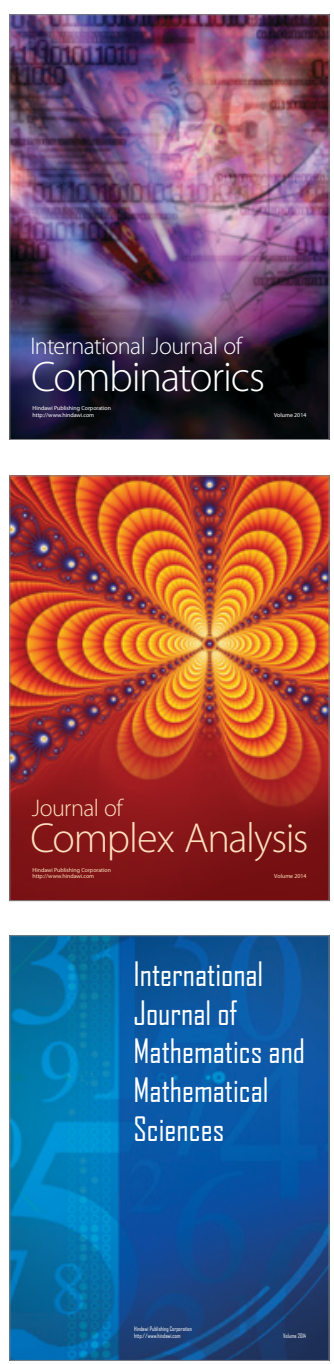
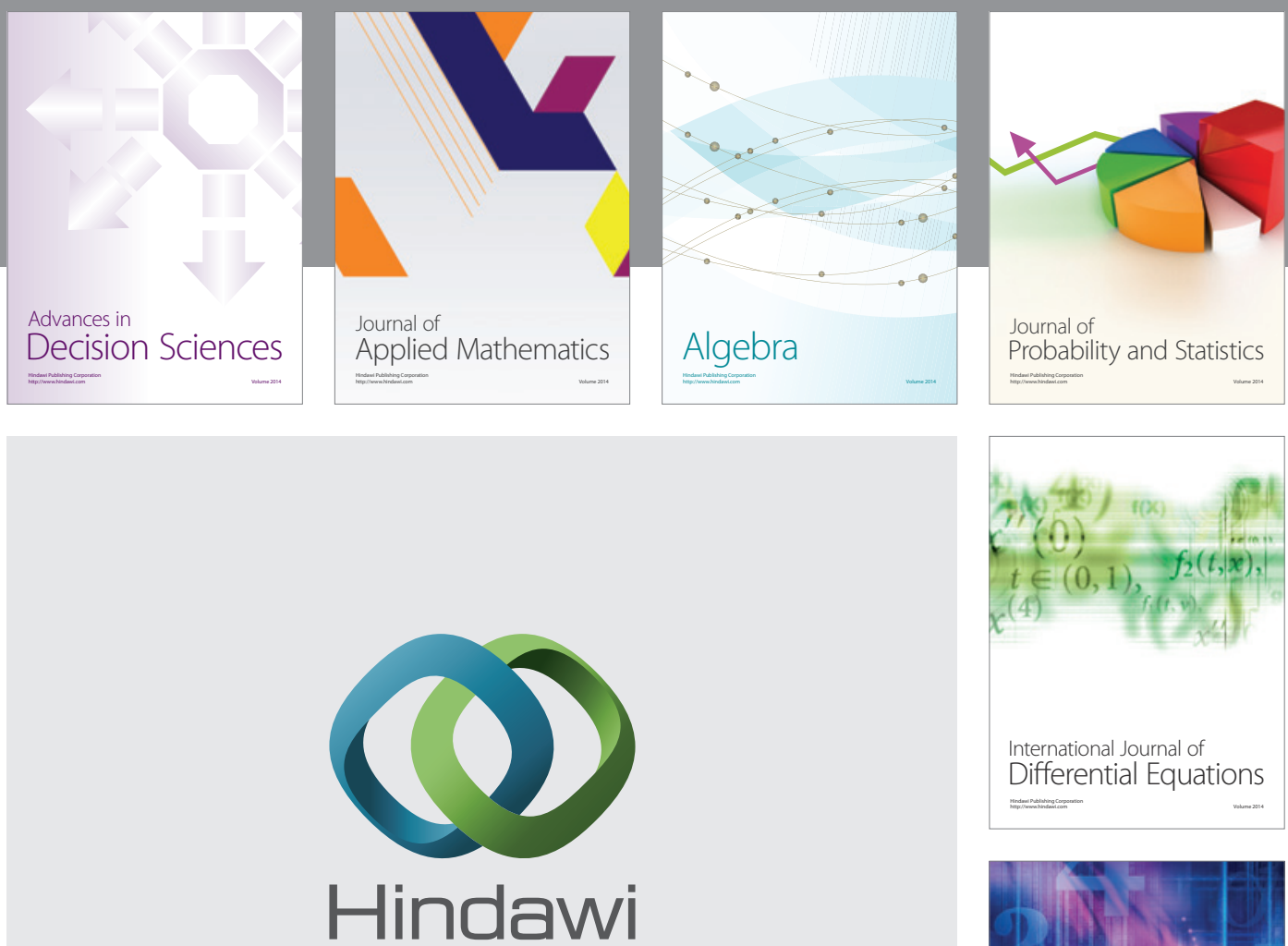

Submit your manuscripts at http://www.hindawi.com
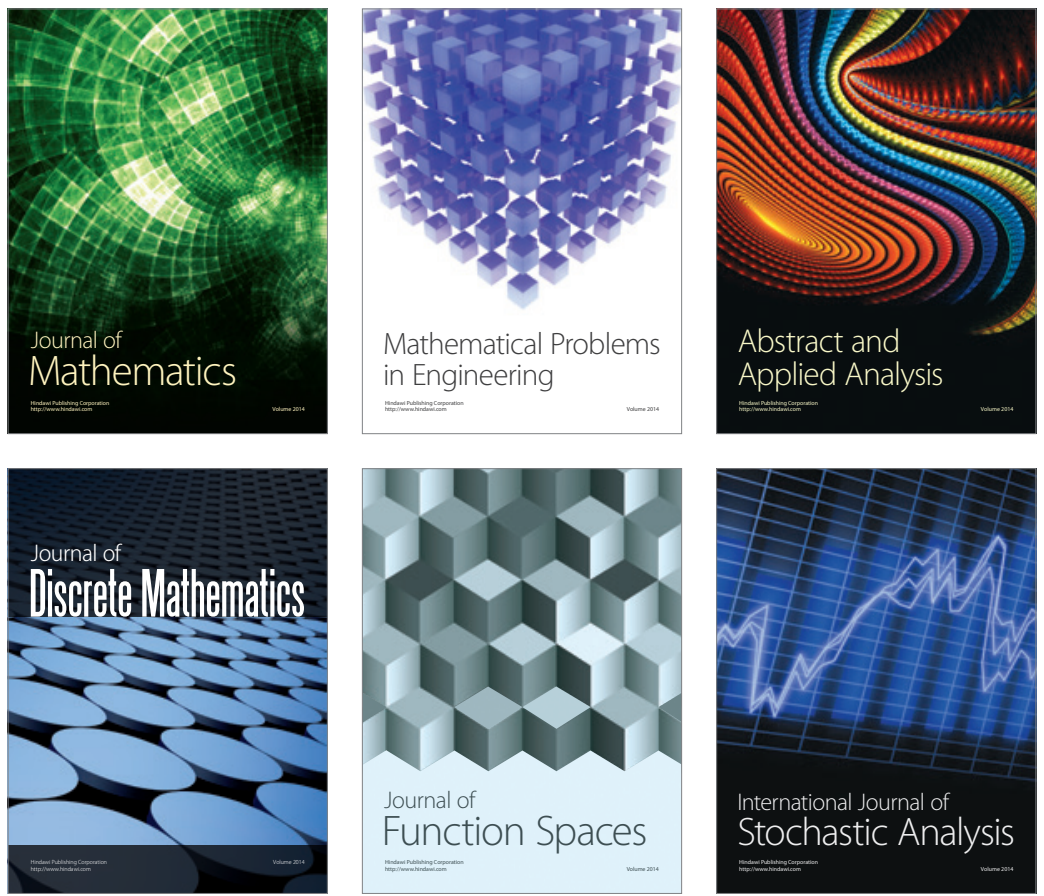

Journal of

Function Spaces

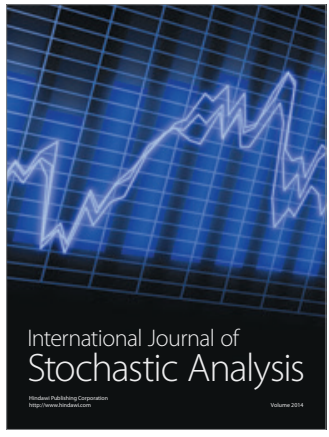

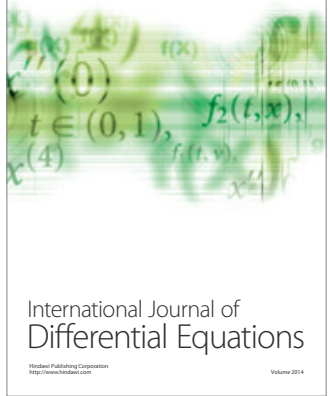
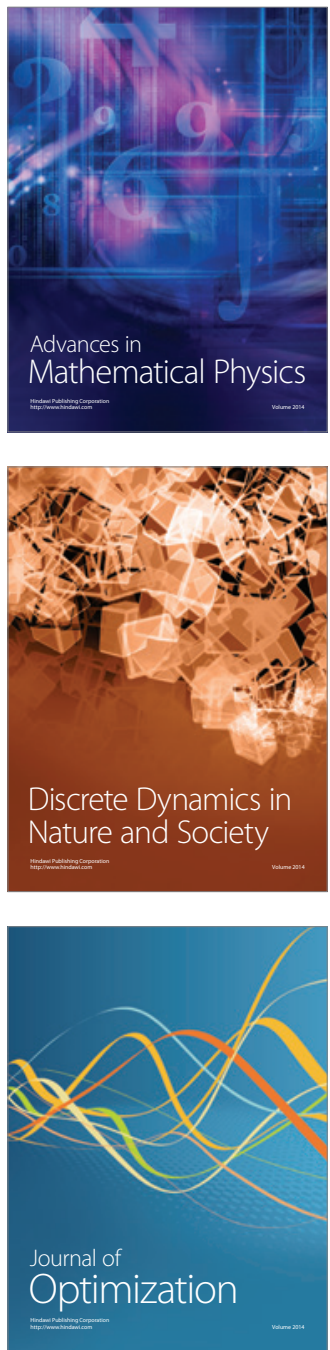